\title{
BMJ Open Maternal night-eating pattern and glucose tolerance during pregnancy: study protocol for a longitudinal study
}

\author{
See Ling Loy, ${ }^{1,2,3}$ Yin Bun Cheung, ${ }^{4,5}$ Mary Chong, ${ }^{3,6}$ \\ Falk Müller-Riemenschneider, ${ }^{6,7}$ Ngee Lek, ${ }^{2,8}$ YS Lee, ${ }^{3,9,10}$ Kok Hian Tan, ${ }^{2,11}$ \\ Bernard Chern, ${ }^{2,12}$ Fabian Yap, ${ }^{2,8,13}$ Jerry Chan ${ }^{1,2}$
}

To cite: Loy SL, Cheung YB, Chong $\mathrm{M}$, et al. Maternal night-eating pattern and glucose tolerance during pregnancy: study protocol for a longitudinal study. BMJ Open 2019:9:e030036. doi:10.1136/ bmjopen-2019-030036

- Prepublication history for this paper is available online. To view these files, please visit the journal online (http://dx.doi org/10.1136/bmjopen-2019030036).

Received 24 February 2019 Revised 02 September 2019 Accepted 17 September 2019

Check for updates

(c) Author(s) (or their employer(s)) 2019. Re-use permitted under CC BY-NC. No commercial re-use. See rights and permissions. Published by BMJ.

For numbered affiliations see end of article.

Correspondence to

Dr See Ling Loy:

Loy.See.Ling@kkh.com.sg

\section{ABSTRACT}

Introduction Coordinating eating schedules with daynight cycles has been shown to improve glucose regulation in adults, but its association with gestational glycaemia is less clear. A better understanding on how eating time can influence glucose levels in pregnancy may improve strategies for gestational glycaemic control. This study aims to examine the association of maternal night-eating pattern with glucose tolerance in the second trimester of pregnancy, and to investigate how lifestyle factors may be related to night-eating pattern.

Methods and analysis This is an observational longitudinal study that targets to recruit 200 pregnant women at 18-24 weeks' gestation from the KK Women's and Children's Hospital in Singapore. Data collection includes sociodemographics, lifestyle habits and obstetric information. Maternal dietary intake is collected using the 4-day food diary and food frequency questionnaire; while 24-hour physical activity, sedentary behaviour, sleep and light exposure are captured using the accelerometer at 18-24 weeks' gestation. Continuous glucose monitoring at 18-24 weeks' gestation, oral glucose tolerance test and insulin test at 24-28 weeks' gestation are performed to assess glycaemic outcomes. Multivariable generalised linear models will be used to analyse the association of maternal night-eating pattern (consumption of meal and snack during 1900-0659 hours) with glycaemic measures, and the associated factors of night-eating pattern, controlling for potential confounders. Recruitment began in March 2019 and is estimated to end in November 2020.

Ethics and dissemination Ethical approval has been granted by the Centralised Institutional Review Board of SingHealth, Singapore (reference 2018/2529). The results will be presented at conferences and disseminated in journal articles.

Trial registration number NCT03803345

\section{INTRODUCTION}

Over time, humans have evolved to keep time with the earth's repeated light-dark cycles. These day-night rhythms orchestrate critical aspects of human physiology, from cell signalling to cellular metabolism; as well as influence habitual aspects of human behaviour, including activity, sleep and energy consumption. ${ }^{1}$ The alignment of eating time with

\section{Strengths and limitations of this study}

- This study will provide information on maternal night-eating pattern during pregnancy and its association with glycaemic outcomes, which will be useful to healthcare professionals and the pregnant population in the effort of glycaemic control.

- This study comprehensively assesses the night-eating pattern, glycaemic profile and lifestyle factors of pregnant women.

- Given the participants are recruited from a single hospital, the sample may not be considered representative of all pregnant women in Singapore.

the body's circadian rhythms, known also as circadian eating, has been shown to improve glucose tolerance, ${ }^{2}$ suggesting that circadian dietary strategies may be a useful way to maintain metabolic health. Pregnant women belong to a high-risk population vulnerable to hyperglycaemia and its consequences. In Singapore, $20 \%$ women develop gestational diabetes mellitus (GDM). ${ }^{3}$ Even at glucose concentrations below the diagnostic cut-off for GDM, risks of adverse perinatal outcomes can occur, and these risks increase continuously in association with rising glucose levels during pregnancy. ${ }^{4}$ Effective interventions to improve glycaemic control in pregnancy are urgently needed.

Although it is known that food quantity and quality influence GDM development, ${ }^{5}$ the effect of circadian eating pattern, ${ }^{6}$ specifically evening meal intake and nocturnal snacking behaviour on glucose regulation in pregnancy, remains an important gap of knowledge. In the general population, late-eating or night-eating has been associated with less healthy eating and more snack intake, ${ }^{7}$ which may be related to metabolic disorders. ${ }^{8}$ It was found that women with GDM were more likely to snack at night compared with those of normal glucose tolerance. ${ }^{9}$ Based on the 
latest nutritional guidelines from the Academy of Nutrition and Dietetics, a new recommendation on meal and snack distribution has been included where women with GDM are encouraged to have three meals and two or more snacks per day. ${ }^{10}$ However, this recommendation did not consider the effect of day-night or circadian cycles, and it was formed based on a consensus approach rather than with supportive evidence.

Therefore, our motivation is to develop an understanding of the role of circadian timing for meals and snacks on blood glucose levels during pregnancy, which is potentially a modifiable behaviour for glycaemic control. The aims of this study are (1) to examine the association of maternal night-eating pattern from the aspect of amount and frequency of meals and snacks with glucose tolerance in the second trimester of pregnancy and (2) to investigate how lifestyle factors, specifically daily physical activity, sedentary behaviour, sleep, diet quality and light exposure may be related to night-eating pattern. These lifestyle factors may influence the association between night-eating pattern and glycaemic measures; yet have not been evaluated previously. The central hypothesis is that small evening meals and less frequent snacking at night are associated with better glucose tolerance at 24-28 weeks' gestation-a period when the screening for GDM is usually done, compared with those with larger evening meals and more frequent snacking at night.

\section{METHODS AND ANALYSIS \\ Study design}

This is an observational longitudinal study, where pregnant women at 18-24 weeks' gestation are recruited and followed until delivery. An overview of the study procedures is illustrated in figure 1.

\section{Participants and recruitment}

This study is conducted at KK Women's and Children's Hospital (KKH), Singapore. KKH houses the largest Obstetrics and Gynaecology department in Singapore, with over $10000(\approx 30 \%)$ live births recorded annually. A non-probability (convenience) sampling method is used to recruit pregnant women who attend scheduled antenatal clinic appointments at KKH. Instead of all antenatal clinics, we only target at one specific clinic with a greater number of potential participants to perform the recruitment due to restricted manpower. We expect the response rate to be $25 \%-35 \%$. Those who meet the selection criteria are invited to participate in this study. Recruitment began in March 2019 and is estimated to end in November 2020.

The sample comprises pregnant women between 18 and 24 weeks' gestation at recruitment, age $\geq 18$ years, who are Singapore citizens or Singapore permanent residents, plan to continue antenatal care at $\mathrm{KKH}$, intend to deliver at KKH and able to provide written informed consent. Excluded women are those with diabetes in pregnancy at recruitment as confirmed by the oral glucose tolerance test (OGTT), have pre-existing type-1 or type-2 diabetes, on routine night-shift work for at least $3 \times$ /week currently or in the last month, use of anticonvulsant medications/ oral steroids currently or in the last month, and with known or suspected allergy to medical grade adhesives. We also exclude pregnant women with chronic kidney disease, preeclampsia and multiple pregnancy due to lack of evidence to support accuracy of using continuous glucose monitoring system (CGMS) (Freestyle Libre Pro, Abbott, Germany) among these patients. Participants who develop a miscarriage or undergo a termination event, unable to comply with the study protocol or wish to discontinue participation are withdrawn from the study.

Recruitment brochures that contain general information of the study are placed in the antenatal clinics. During the recruitment process, trained research staff inform potential women of the study both verbally and with written information. Women who are agreeable to participate provide written informed consent. Those who decline to participate continue to receive their hospital antenatal care as usual, and care provided to each pregnant woman is not affected nor influenced by the woman's decision to either participate or not participate in the study.

\section{Study procedures}

Study visits (recruitment and follow-up visits) of this study are determined based on maternal antenatal

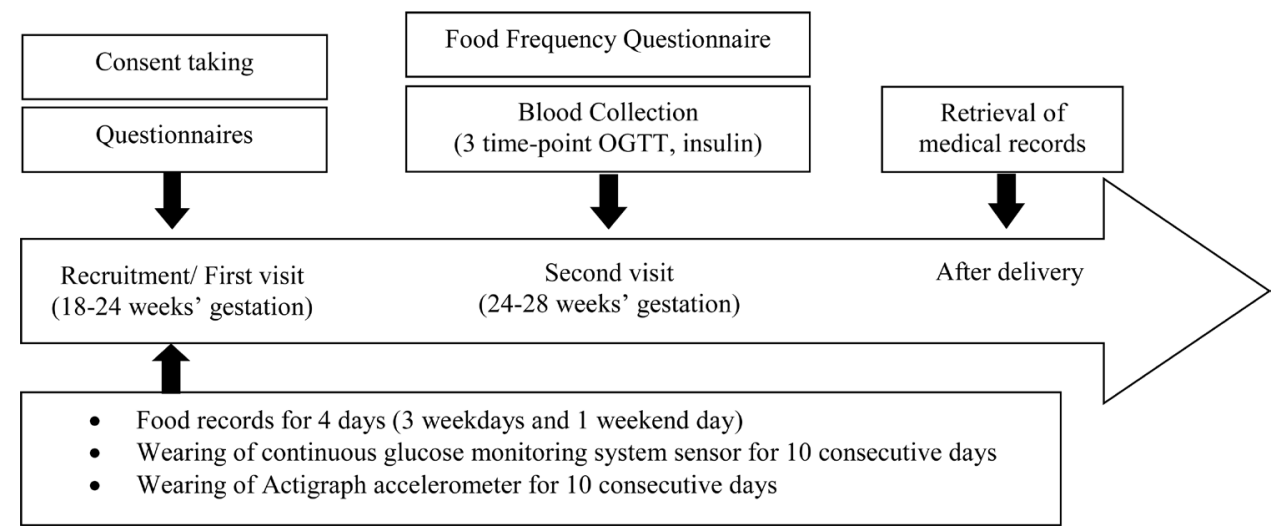

Figure 1 Flow diagram of the study design. OGTT, oralglucose tolerance test. 
appointment schedules. After providing written informed consent at the recruitment visit (18-24 weeks' gestation), enrolled participants are interviewed for information on sociodemographics and lifestyle habits. At the same visit, participants are provided with a food diary to record 4-day dietary intake. All participants are also asked to wear a CGMS sensor on the back of their upper arm to measure 24-hour glucose levels over 10 consecutive days, and an accelerometer on the wrist to capture their 24-hour physical activity pattern, sedentary behaviour, sleep and light exposure over 10 consecutive days.

At 24-28 weeks' gestation, participants undergo a $75 \mathrm{~g}$ OGTT (0,60 and $120 \mathrm{~min})$ along with fasting insulin test. During the same period, research staff conduct an interviewer-administered online food frequency questionnaire (FFQ) in the antenatal clinic to assess maternal food intake over the last 1 month. After delivery, research staff retrieve medical notes to document obstetric outcomes.

\section{Sample size}

The sample size was calculated based on estimate of correlation coefficient between maternal circadian eating time and plasma glucose at mid/late pregnancy from previous studies. ${ }^{6}{ }^{11}$ Based on two-sided significance level set at $5 \%$ and with $80 \%$ power, 123 pregnant women are required to detect a minimum correlation coefficient of 0.25 between night-time caloric intake and plasma glucose. Assuming that variance inflation factor arising from covariate adjustment is 1.5 and with a dropout rate of $10 \%$, the total sample size required for primary aim is 200 pregnant women.

\section{Study measurements}

Baseline sociodemographic information and potential confounding variables are collected through questionnaires at visit 1 . These include age, ethnicity (Chinese, Malay, Indian, others), education (none, primary, secondary, tertiary), occupation (unemployed, employed), smoking status (never, past smoker, active smoker, passive smoker), alcohol consumption (never, monthly, weekly, daily), nausea/ vomiting (no, moderate, severe, very severe), meal regularity, electronic media use before bedtime and mood. Health and obstetric histories are obtained from the electronic medical notes. Table 1 shows the details of the types of data that are collected in this study.

\section{Exposure measures}

Night-eating pattern

At visit 1 , the research staff guide the participants to fill out the 4-day food diary ( 3 weekdays and 1 weekend day). Participants are required to record the time, type, description and amount of food and beverages consumed throughout the day. Pictures of household measuring utensils and various food portion sizes are printed in the food diary to assist participants in quantifying their food intake. In the case that food diary is not able to be filled up by the participant, research staff conduct 24-hour recall for dietary data collection through phone interview.

Nutrient analysis of dietary records will be performed using the Dietplan (Forestfield Software, UK), which contains a local food composition database. Based on the evidence showing that sunlight is a strong environmental signal for the human circadian clock, ${ }^{12}$ we determine daytime and night-time periods according to the local time of sunrise ( $\sim 700$ hours) and sunset ( 1900 hours $),{ }^{6}$ which are relatively consistent throughout the year given Singapore's equatorial position $\left(1.3^{\circ} \mathrm{N}, 103.8^{\circ} \mathrm{E}\right) .{ }^{13}$ With that, night-eating pattern will be assessed based on the amount and frequency of meals and snacks during 1900-0659 hour.

\section{Diet quality}

Diet quality will be derived from a 125 food items electronic graphic FFQ at visit 2, where the Healthy Eating Index will be calculated. This FFQ is adapted from the paper-based FFQ used by the National Nutrition Survey 2010. ${ }^{14}$ Participants are required to indicate frequency of foods consumed in the last 1 month, by selecting one out of six frequency options ranging from ' $1-3$ times per month' to '2-3 times per day'. Individual portion size is asked for each food, and pictures of the various portion sizes are provided for more accurate quantification.

\section{Physical activity, sedentary behaviour, sleep and light exposure}

The Actigraph wGT3X-BT (Actigraph LLC, Pensacola, Florida, USA) is used to objectively monitor 24-hour physical activity, sedentary behaviour, sleep and light exposure. ${ }^{1516}$ The wGT3X-BT is a triaxial accelerometer designed to record continuous high-resolution physical activity and sleep/wake information. It includes an integrated ambient light sensor that delivers lux values alongside activity information. Lux is a measure of light intensity. At visit 1, participants wear the device on their non-dominant wrist for 10 consecutive days. The device does not have to be removed during aquatic activities or showering. An information sheet describing how to wear the device correctly is provided. The actigraphy data will be downloaded using the ActiLife software and processed using the R package GGIR. ${ }^{17}$ Variables such as energy expenditure (MET-min/day), sleep/ wake parameters (total sleep time, total wake time and number of awakenings) and amount of light exposure (Lux) will be derived from the actigraphy data.

Questionnaires on physical activity, sedentary behaviour, sleep and light exposure are also administered at the same visit. Participants are interviewed using the modified International Physical Activity Questionnaire-Short Form (IPAQ-SF) to self-report their physical activity in the last 7 days. ${ }^{18}$ The modified questionnaire evaluates the vigorous physical activity, the moderate physical activity and the walking time. We removed question asking about the sitting time from the original IPAQ-SF and included it in the questionnaire used to 


\begin{tabular}{|c|c|c|c|}
\hline Data & Visit 1 (18-24 weeks' gestation) & Visit 2 (24-28 weeks' gestation) & After delivery \\
\hline Informed consent & $\sqrt{ }$ & & \\
\hline Eligibility criteria & $\sqrt{ }$ & & \\
\hline \multicolumn{4}{|l|}{ Baseline characteristics } \\
\hline Educational attainment & $\sqrt{ }$ & & \\
\hline Occupation & $\sqrt{ }$ & & \\
\hline Ethnicity & $\sqrt{ }$ & & \\
\hline Prepregnancy body mass index & $\sqrt{ }$ & & \\
\hline Smoking status & $\sqrt{ }$ & & \\
\hline Alcohol intake & $\sqrt{ }$ & & \\
\hline Nausea/vomiting & $\sqrt{ }$ & & \\
\hline \multicolumn{4}{|l|}{ Questionnaires } \\
\hline Physical activity & $\sqrt{ }$ & & \\
\hline Sedentary behaviour & $\sqrt{ }$ & & \\
\hline Sleep habit & $\sqrt{ }$ & & \\
\hline Light exposure & $\sqrt{ }$ & & \\
\hline Electronic media use before bedtime & $\sqrt{ }$ & & \\
\hline Mood & $\sqrt{ }$ & & \\
\hline Actigraphy monitoring & $\sqrt{ }$ & & \\
\hline \multicolumn{4}{|l|}{ Diet } \\
\hline Meal regularity & $\sqrt{ }$ & & \\
\hline Food diary & $\sqrt{ }$ & & \\
\hline Food frequency questionnaire & & $\sqrt{ }$ & \\
\hline \multicolumn{4}{|l|}{ Glycemic measures } \\
\hline Continuous glucose monitoring & $\sqrt{ }$ & & \\
\hline Oral glucose tolerance test & & $\sqrt{ }$ & \\
\hline Fasting insulin test & & $\sqrt{ }$ & \\
\hline \multicolumn{4}{|l|}{ Obstetric information } \\
\hline Gestational weight gain & & & $\sqrt{ }$ \\
\hline Obstetric history & & & $\sqrt{ }$ \\
\hline Delivery outcomes & & & $\sqrt{ }$ \\
\hline Pregnancy complications & & & $\sqrt{ }$ \\
\hline Birth outcomes & & & $\sqrt{ }$ \\
\hline
\end{tabular}

assess sedentary behaviour. The data will be computed in metabolic equivalents (MET-min/week) scores. Questionnaire on sedentary behaviour which is modified from the Adult Sedentary Behaviour Questionnaire is performed. ${ }^{19}$ The questionnaire evaluates time spent sedentary in the last 7 days, including sitting time at work, sitting/lying down time to watch television, to use electronic devices at mealtimes, while driving or reading. Participants also self-administer the Pittsburgh Sleep Quality Index questionnaire to assess their sleep habits in the last month, ${ }^{20}$ and the Harvard Light Exposure Assessment questionnaire to assess their main light sources exposure in hourly basis on a typical weekday and weekend day. ${ }^{21}$

\section{Outcomes measures}

The main outcome of this study is plasma glucose levels as assessed by OGTT after visit 1 , routinely between 24 and 28 weeks' gestation. The secondary outcomes include glycaemic variability based on continuous glucose monitoring profile, insulin level, GDM development, gestational weight gain (GWG), delivery and birth outcomes.

\section{OGTT and insulin test}

Participants undergo a 75-g OGTT after an overnight fast of 8-10 hours at visit 2. This is a routine universal test for all pregnancies at KKH. The procedures of fasting and OGTT are explained by the research staff and nurses in the antenatal clinic before visit 2. Venous fasting plasma 
glucose and insulin, 1-hour and 2-hour postload plasma glucose levels are measured in the KKH lab. The participants are informed of their OGTT results by the attending doctors during their subsequent antenatal visits. Any abnormal findings are treated as per clinical practice. GDM is defined according to the WHO 2013 criteria. ${ }^{22}$

\section{Continuous glucose monitoring}

A 10-day continuous glucose monitoring for assessment of glycaemic variability is initiated at visit 1 by using the FreeStyle Libre Pro Flash Glucose Monitoring System (Abbott, Germany). The CGMS sensor is applied on the back of upper arm. No calibration for the sensor is required throughout the 10-day period. Readings from the CGMS are unavailable to participants in real time to avoid bias that may arise from unmasked, real-time glucose readings. We do not perform this procedure at visit 2 as if the participant is diagnosed with GDM, they will receive dietary counselling and/or insulin treatment which can alter the CGMS readings.

\section{Gestational weight gain}

Research staff retrieve maternal weight at every antenatal visit from the medical notes after delivery. Total and rate of GWG (GWG per week) will be computed. Classification of GWG will be performed according to the Institute of Medicine's guidelines. ${ }^{23}$

\section{Delivery and birth outcomes}

Research staff retrieve information on delivery and birth outcomes from the medical notes after delivery.

\section{Statistical analysis}

We will perform statistical analyses using the SPSS statistical package or Stata Statistical Software. Multivariable generalised linear models will be used to examine the associations of maternal night-eating pattern (eg, amount of last meal consumption in $\mathrm{kcal}$, number of nightly snacking episodes) with glycaemic measures, GWG and obstetric outcomes, adjusting for potential covariates. We will define night-time based on the period between 1900 and 0659 hours (from sunset to sunrise) as described above. We will also perform additional analysis to further define night-time based on different criteria (eg, after 20:00 or 21:00) to explore result differences.

Selection of covariates will be determined from literature review, directed acyclic graph and/or observed statistical significance associations with exposures and outcomes. In view of the relation between night-fasting and plasma glucose as reported previously, ${ }^{6}$ effect of night-fasting duration will therefore be considered and adjusted in the model. Multivariable generalised linear models will also be used to examine associations of physical activity, sedentary behaviour, sleep, diet quality and light exposure with night-eating pattern, controlling for potential covariates.

We will conduct stratified analyses to assess potential effect modification by maternal age and prepregnancy body mass index. We will evaluate the significance of effect modification on the multiplicative scale by including an interaction term (night-eating pattern $\times$ age or nighteating pattern $\times$ prepregnancy body mass index) in the model.

We will impute missing data using multiple imputation analyses by chained equations. ${ }^{24}$ The number of imputations will be determined based on percentage of missing values $^{25}$ and results of total imputations will be pooled using Rubin's rule. ${ }^{26}$ To evaluate whether the imputation of missing data may have affected the results, we will perform sensitivity analyses on participants with complete data.

\section{Quality control}

The research staff received training on how to perform study procedures, including administration of questionnaires, food diary and FFQ handling of CGMS device and accelerometer. The research staff were required to complete the competency assessments to ensure data quality before conducting the procedures in this study. Monthly meetings are held with the principal investigator to review study procedures and data collected. An annual report on study progress will be prepared.

\section{Data monitoring and management}

Participants are anonymised and assigned with a specific ID at study entry. Data are managed using the Research Electronic Data Capture electronic data capture tool. To ensure accuracy and completeness of data entry, data are checked by identifying if there is any outlier or missing value. The data checking process is performed in the first 3 months of the study and so on, such that the experience gained can be used to train the research staff for improvement. Paper documents are kept in a locked cabinet and electronic data are stored on password-protected computers or hard-disk drives which can only be accessed by research team members. All records will be kept for at least 6 years after completing the study.

\section{Patient and public involvement}

The research questions, exposure and outcome measures were determined based on the evaluation of knowledge gap as identified from literature review, and through discussions with clinicians, researchers and healthcare staff who have been involved in maternal child care. Although participants did not directly contribute to the development of research questions and the study design, their needs and preferences were considered throughout the process. Participants will be informed for their blood test results. Findings of the study will be disseminated to participants at their request.

\section{ETHICS AND DISSEMINATION}

Participants sign a written informed consent and are provided with written information about the study. This study is conducted according to the Helsinki Declaration. When there are any changes in the study protocol or instruments used during the study period, further 
ethical approval is sought, follow by reconsenting the participants whenever necessary. Previously collected data which are not able to be matched with the current data as collected using the latest revised version will be removed and treated as missing variable, if data recollection is not possible. This study has been registered at ClinicalTrials.gov. Findings of the study will be presented at conferences and disseminated in peer-reviewed journals. Media releases will be considered to maximise visibility of the findings to the general public.

\section{DISCUSSION}

This protocol outlines the rationale and design of an observational longitudinal study that aims to examine the associations of night-eating pattern with glycaemic measures and obstetric outcomes among pregnant women in Singapore. Lifestyle factors associated with night-eating pattern are evaluated. Data from this study will contribute to narrow the gap in knowledge related to maternal night-eating pattern during pregnancy, which has received relatively less attention in the literature compared with general adult population.

The strengths of the study include comprehensive assessment of maternal diet using 4-day food diary and FFQ providing a representative estimate of habitual dietary intake. The use of accelerometer allows detailed investigations and objective measures for physical activity, sedentary behaviour, sleep and light exposure, to enhance data accuracy. Other than using OGTT and insulin response as the glycaemic outcomes, this study also describes maternal glycaemic variability based on continuous glucose monitoring profile, giving us the opportunity to understand the gestational glucose patterns which may independently contribute to GDM-related complications. ${ }^{27}$

This study may be limited by its external validity as it only includes participants from one hospital in Singapore. The use of non-probability sampling method to recruit participants may introduce selection bias; however, this is restricted by the practical and feasible recruitment mechanism at the study site. Therefore, caution is required to extrapolate the findings to general pregnant population. Nevertheless, KKH houses the largest public maternity unit in Singapore, and manages $\sim 30 \%$ of all live births in Singapore, across a wide sociodemographic spectrum. To check for generalisability of findings, we will explore for differences by comparing basic demographic data obtained from this study with data available from other studies involving larger population of pregnant women in Singapore. ${ }^{28}$

This study aims to serve as a baseline reference for planning interventional clinical trial to examine the effect of aligning eating time with day-night cycles on glucose regulation and GDM risk in pregnancy. This may help to develop evidence-based recommendations on maternal nutrition related to meal and snack distribution, in order to improve gestational glycaemic control, reduce the risk of GDM, and thus improving pregnancy and childhood outcomes. Also, this study may have public health implications as night-eating has become a common practice and habit among urban communities.

\section{Author affiliations}

${ }^{1}$ Department of Reproductive Medicine, KK Women's and Children's Hospital, Singapore, Singapore

${ }^{2}$ Duke-NUS Medical School, 8 College Road, Singapore, Singapore

${ }^{3}$ Singapore Institute for Clinical Sciences, Agency for Science, Technology and Research (A*STAR), Singapore, Singapore

${ }^{4}$ Programme in Health Services \& Systems Research and Center for Quantitative

Medicine, Duke-NUS Medical School, Singapore, Singapore

${ }^{5}$ Center for Child Health Research, Tampere University, Tampere, Finland

${ }^{6}$ Saw Swee Hock School of Public Health, National University of Singapore,

Singapore, Singapore

${ }^{7}$ Institute of Social Medicine, Epidemiology and Health Economics, Charité University

Medical Centre Berlin, Berlin, Germany

${ }^{8}$ Department of Paediatrics, KK Women's and Children's Hospital, Singapore, Singapore

${ }^{9}$ Department of Paediatrics, Yong Loo Lin School of Medicine, National University of Singapore, National University Health System, Singapore, Singapore

${ }^{10}$ Division of Paediatric Endocrinology, Khoo Teck Puat-National University Children's Medical Institute, National University Hospital, National University Health System,

Singapore, Singapore

${ }^{11}$ Department of Maternal Fetal Medicine, KK Women's and Children's Hospital, Singapore, Singapore

${ }^{12}$ Department of Obstetrics \& Gynaecology, KK Women's and Children's Hospital, Singapore, Singapore

${ }^{13}$ Lee Kong Chian School of Medicine, Nanyang Technological University, Singapore, Singapore

Acknowledgements We gratefully acknowledge the contribution of research coordinator, Dora Xin Ping Gan and research administrator, Jinjie Lin, to the planning of this study.

Contributors SLL is the principal investigator of the study, along with $\mathrm{FY}, \mathrm{YBC}$, $\mathrm{MC}, \mathrm{FM}-\mathrm{R}, \mathrm{NL}, \mathrm{YSL}, \mathrm{KHT}$ and $\mathrm{BC}$ as coinvestigators who have contributed to the conception and design of the study. SLL, FY and JC assisted in the development and implementation of the study. SLL drafted the manuscript. SLL, YBC, MC, FM-R, NL, YSL, KHT and FY commented, edited and revised the manuscript. All authors read and approved the final manuscript.

Funding This research is supported by the Singapore Ministry of Health's National Medical Research Council under its Open Fund-Young Individual Research Grant (NMRC/OFYIRG/0082/2018).

Competing interests None declared.

Patient consent for publication Not required.

Ethics approval Ethical approval has been granted by the Centralised Institutional Review Board of SingHealth (reference 2018/2529).

Provenance and peer review Not commissioned; externally peer reviewed.

Open access This is an open access article distributed in accordance with the Creative Commons Attribution Non Commercial (CC BY-NC 4.0) license, which permits others to distribute, remix, adapt, build upon this work non-commercially, and license their derivative works on different terms, provided the original work is properly cited, appropriate credit is given, any changes made indicated, and the use is non-commercial. See: http://creativecommons.org/licenses/by-nc/4.0/.

\section{REFERENCES}

1 Johnston JD, Ordovás JM, Scheer FA, et al. Circadian rhythms, metabolism, and Chrononutrition in rodents and humans. Adv Nutr 2016;7:399-406.

2 Rothschild J, Hoddy KK, Jambazian P, et al. Time-restricted feeding and risk of metabolic disease: a review of human and animal studies. Nutr Rev 2014;72:308-18.

3 Chong Y-S, Cai S, Lin H, et al. Ethnic differences translate to inadequacy of high-risk screening for gestational diabetes mellitus 
in an Asian population: a cohort study. BMC Pregnancy Childbirth 2014; $14: 345$

4 Metzger BE, Lowe LP, Dyer AR, et al. Hyperglycemia and adverse pregnancy outcomes. N Engl J Med 2008;358:1991-2002.

5 Schoenaker DAJM, Mishra GD, Callaway LK, et al. The role of energy, nutrients, foods, and dietary patterns in the development of gestational diabetes mellitus: a systematic review of observational studies. Diabetes Care 2016;39:16-23.

6 Loy SL, Chan JKY, Wee PH, et al. Maternal circadian eating time and frequency are associated with blood glucose concentrations during pregnancy. J Nutr 2017;147:70-7.

7 Gallant A, Lundgren J, Drapeau V. Nutritional aspects of late eating and night eating. Curr Obes Rep 2014;3:101-7.

8 Oike H, Oishi K, Kobori M. Nutrients, clock genes, and Chrononutrition. Curr Nutr Rep 2014:3:204-12.

9 Park $\mathrm{H}$-jin, Lee J, Kim J-M, et al. A study of snack consumption, night-eating habits, and nutrient intake in gestational diabetes mellitus. Clin Nutr Res 2013;2:42-51.

10 Duarte-Gardea MO, Gonzales-Pacheco DM, Reader DM, et al. Academy of nutrition and dietetics gestational diabetes evidence-based nutrition practice guideline. J Acad Nutr Diet 2018;118:1719-42.

11 Chandler-Laney PC, Schneider CR, Gower BA, et al. Association of late-night carbohydrate intake with glucose tolerance among pregnant African American women. Matern Child Nutr 2016;12:688-98.

12 Wright KP, McHill AW, Birks BR, et al. Entrainment of the human circadian clock to the natural light-dark cycle. Curr Biol 2013;23:1554-8.

13 Astronomical Applications Department. Sun or moon rise/set table for one year. Washington, DC: U.S. Naval Observatory, 2016. http:// aa.usno.navy.mil/data/docs/RS_OneYear.php\#formb

14 Health Promotion Board Singapore. Report of the National nutrition survey 2010. Available: https://www.hpb.gov.sg/docs/default-source/ pdf/nns-2010-report.pdf?sfvrsn=18e3f172_2 [Accessed cited 4 Jan 2019].

15 Aggio D, Smith L, Fisher A, et al. Association of light exposure on physical activity and sedentary time in young people. Int J Environ Res Public Health 2015;12:2941-9.

16 Migueles JH, Cadenas-Sanchez C, Ekelund U, et al. Accelerometer data collection and processing criteria to assess physical activity and other outcomes: a systematic review and practical considerations. Sports Med 2017;47:1821-45

17 van Hees VT, Fang Z, Zhao JH, et al. Package 'GGIR': Raw Accelerometer Data Analysis, 2018. Available: https://cran. r-project.org/web/packages/GGIR/GGIR.pdf [Accessed 10 Jan 2019].

18 IPAQ research committee. Guidelines for data processing and analysis of the International physical activity questionnaire (IPAQ) 2005. Available: http://www.institutferran.org/documentos/ scoring short_ipaq_april04.pdf [Accessed 18 Dec 2018].

19 AHY C, SHX N, Koh D, et al. Domain-Specific adult sedentary behaviour questionnaire (ASBQ) and the GPAQ single-item question: a reliability and validity study in an Asian population. Int $J$ Environ Res Public Health 2018;15.

20 Buysse DJ, Reynolds CF, Monk TH, et al. The Pittsburgh Sleep Quality Index: a new instrument for psychiatric practice and research. Psychiatry Res 1989;28:193-213.

21 Bajaj A, Rosner B, Lockley SW, et al. Validation of a light questionnaire with real-life photopic illuminance measurements: the Harvard light exposure assessment questionnaire. Cancer Epidemiol Biomarkers Prev 2011;20:1341-9.

22 World Health Organization. Diagnostic criteria and classification of hyperglycaemia first detected in pregnancy: a World Health Organization guideline. Diabetes Res Clin Pract 2014;103:341-63.

23 IOM (Institute of Medicine) and NRC (National Research Council). Weight gain during pregnancy: reexamining the guidelines. Washington, DC: National Academies Press, 2009.

24 Royston P. Multiple imputation of missing values. Stata J 2004;4:227-41.

25 Cheung YB. Analysis of repeated measurements and clustered data. In: Statistical analysis of human growth and development. USA, Boca Raton (FL): CRC Press, 2014

26 Rubin DB. Multiple imputation for nonresponse in surveys. USA, New York: John Wiley \& Sons, 2004.

27 Law GR, Ellison GTH, Secher AL, et al. Analysis of continuous glucose monitoring in pregnant women with diabetes: distinct temporal patterns of glucose associated with Large-for-GestationalAge infants. Diabetes Care 2015;38:1319-25.

28 Soh S-E, Tint MT, Gluckman PD, et al. Cohort profile: Growing Up in Singapore Towards healthy Outcomes (GUSTO) birth cohort study. Int J Epidemiol 2014;43:1401-9. 